http://www.jfas.info

\title{
VOICE OVER IP USING SIP SERVER: WIDE AREA NETWORK PERFORMANCE
}

\section{ANALYSIS}

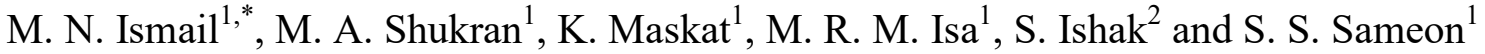 \\ ${ }^{1}$ Faculty of Defense Science and Technology, National Defence University of Malaysia, \\ Sungai Besi, 57000 Kuala Lumpur, Malaysia \\ ${ }^{2}$ College of Information Technology, UniversitiTenagaNasional, 43000 Kajang, Selangor, \\ Malaysia
}

Published online: 10 September 2017

\begin{abstract}
In this project, we propose the use of VoIP application as an alternative for making audio and video conference instead of using the normal telephone line. This technology facilitates the users in the aspect of cost saving as the conference can be made, as long as the users have the access to the internet without having to be charged with any additional charges. The objective of carrying out this project is to test the performance of the codecs installed in the softphone software based on jitter, mean opinion score (MOS), delay and packet loss. The performance of the codecs is analyzed by using network performance monitoring tool available these day. Based on the result, we suggest the best codecs to be used for the software that can guarantee the best performance for the user.
\end{abstract}

Keywords: delay; jitter; voice over IP; VoIP.

Author Correspondence, e-mail: m.nazri@upnm.edu.my

doi: http://dx.doi.org/10.4314/jfas.v9i3s.50 


\section{INTRODUCTION}

In this project, we are using Linphone softphone as our VoIP application software. Usually, we need to purchase a SIP account from a VoIP operator in order to use any softphone software but Linphone offers their own and free SIP service. The VoIP operator's role is to provide gateways that allows the users to make call. Linphone is a general purpose SIP softphone that is not bound to any operator. Because it is compatible with SIP, it can work with any VoIP operator using SIP (most of them use SIP, the most notable exception being Skype). Linphone.org is not affiliated nor recommends a VoIP operator in particular.

In addition, we need to observe the performance of connection occurred in the softphone in terms of packet loss, jitter, latency and mean opinion score (MOS). So, we are using PRTG as network monitoring software. PRTG Network Monitor runs within our network, collecting various statistics from the machines, software and devices which we already designate. With PRTG, we can easily share data performance via live graphs and custom reports. We also used VoIP Spear, a call quality monitoring service to monitoring the performance of call conference we made among the group members. VoIP Spear is a global VoIP monitoring service provider with strategically located testing servers in North America, Europe, Asia and South America. Get the most accurate VoIP call quality profile through location-based call monitoring.

Furthermore, we are using VisualRoute, an online traceroute tool to trace the server from Singapore which is used by VoIP Spear since Malaysia does not have server that support VoIP Spear. The server from Singapore sent the target information to TM Malaysia through the user IP.

Voice over IP is a reality nowadays, every day more and more people use this system to phone around all the world. There are many common programs which make it easy to use VoIP: Skype, MSN messenger, VoIPcheap, VoIP-buster, etc. [1]. Today, many researches concentrate on wireless technology implementation on VoIP service. In the digital era, the increase of network bandwidth and the ubiquitous wireless access facilitates the creation of more and more innovative network services. Among these services, Voice over Internet Protocol (VoIP) is surely one of the most popular and successful real-time multimedia services on the Internet [2]. 
Today, many organizations are using WLANs as a medium for communication, so it is important to investigate how VoIP over WLAN performs based on previous study [3]. Wireless VoIP applications make the very inefficient use of WLAN resources. Due to the large overhead involved in transmitting small packets in an 802.11 WLAN, the bandwidth available for VoIP traffic is far less than its maximal $11 \mathrm{Mbps}$ data rate it currently supports [4].

There has been much activity in the area of WLAN performance analysis in the last few years. The most relevant related works are highlighted here. Previous studies did their work in this area by developing the analytical model [5-7] to compute the saturation throughput of the Distributed Coordination Function (DCF) scheme. Other 802.11 related researches have focused on approaches to adapting system parameters [8-10].

\section{RESULTS AND DISCUSSION}

This section has shown the development, analyzing, measuring of SIP server performance over wide area network. The SIP server located at Singapore and VoIP client located in Malaysia. The VoIP communication occurs between Malaysia and Singapore. Linphone tool is used as a softphone. Fig. 1 shows the interface of Linphone setting and parameters. Fig. 2 and Fig. 3 show the VoIP packet communication between two parties using GSM and G.711 voice codecs.

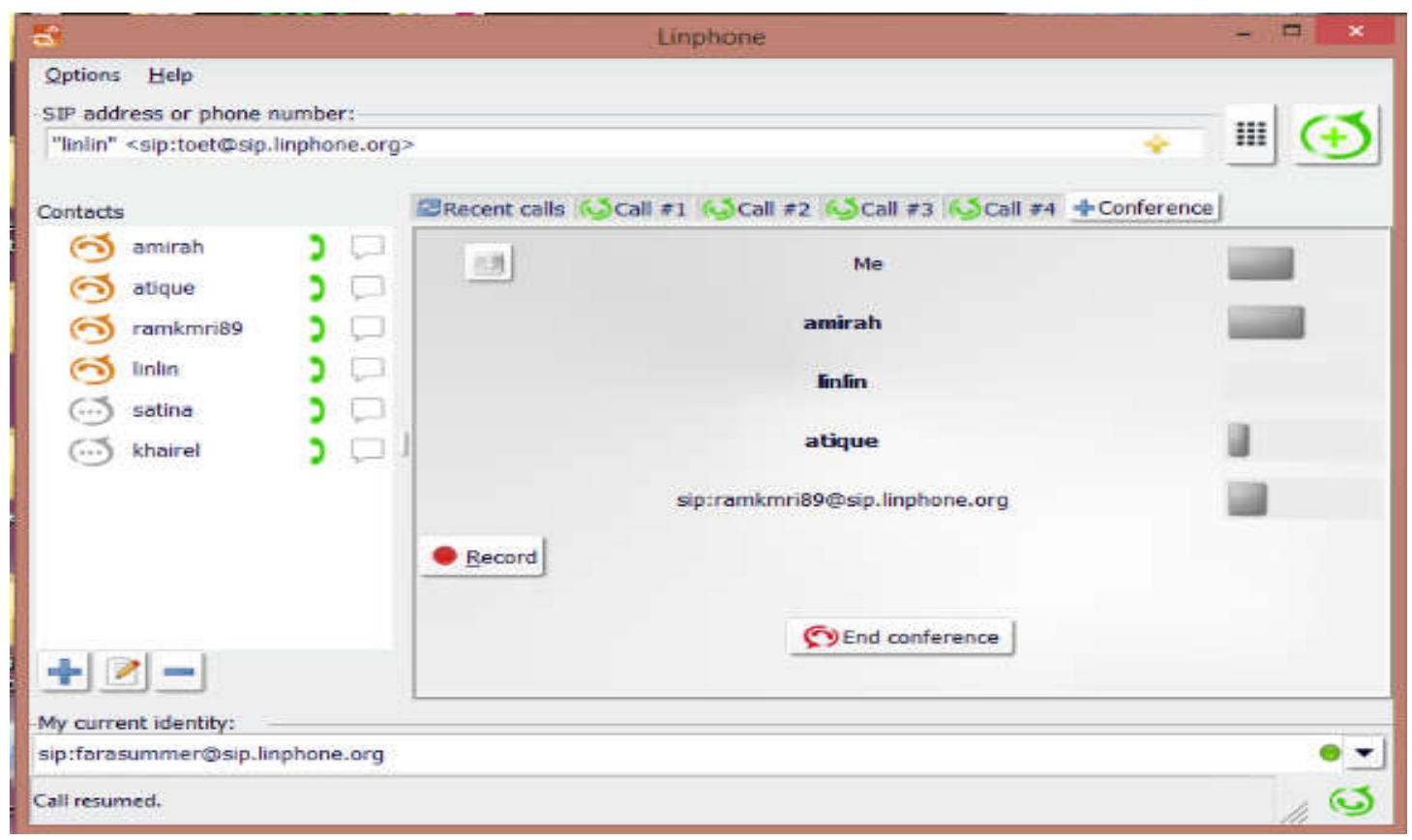

Fig.1.Linphone interface and parameters 


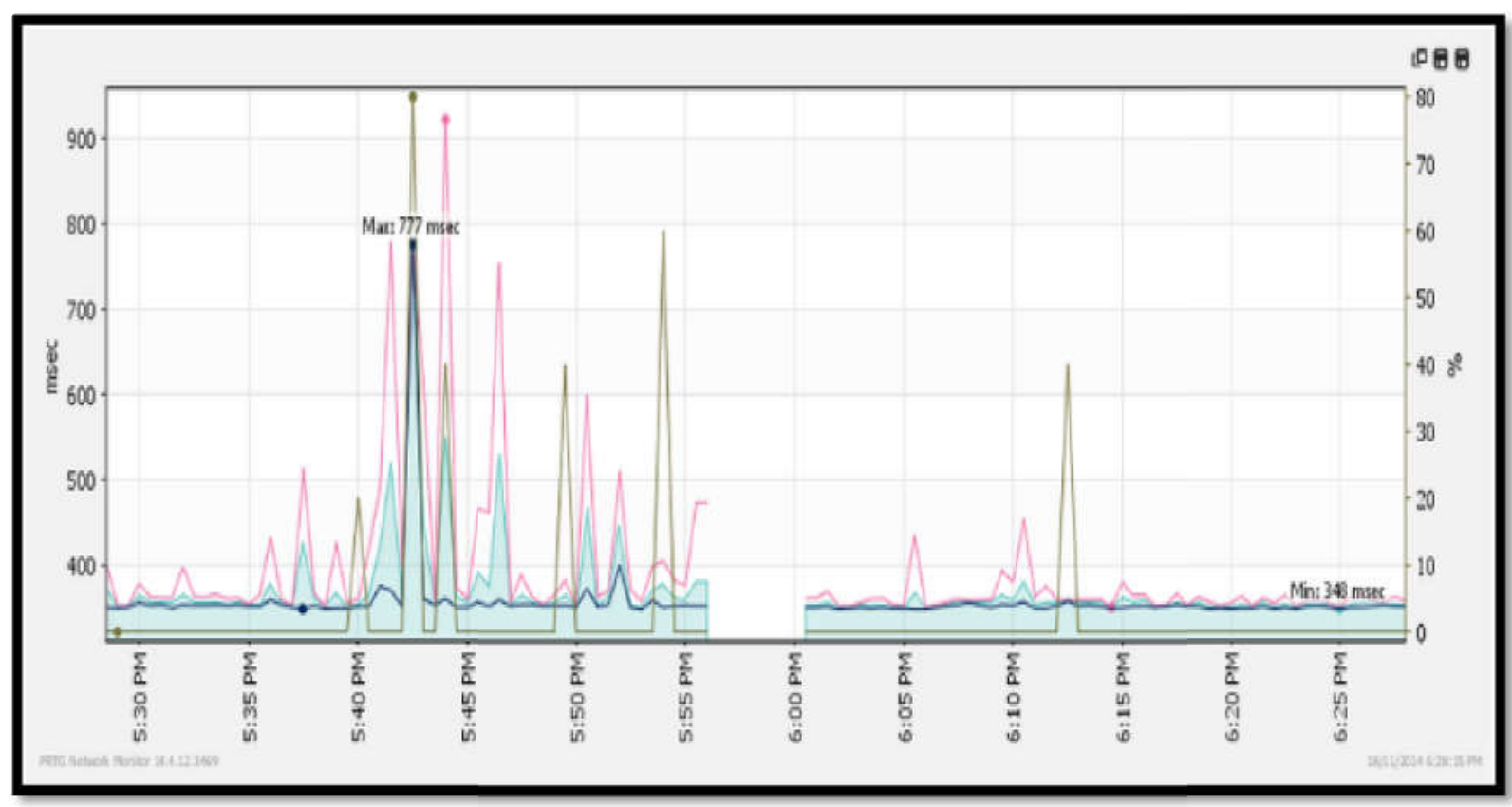

Fig.2. VoIP packet transmission using G.711 Codec

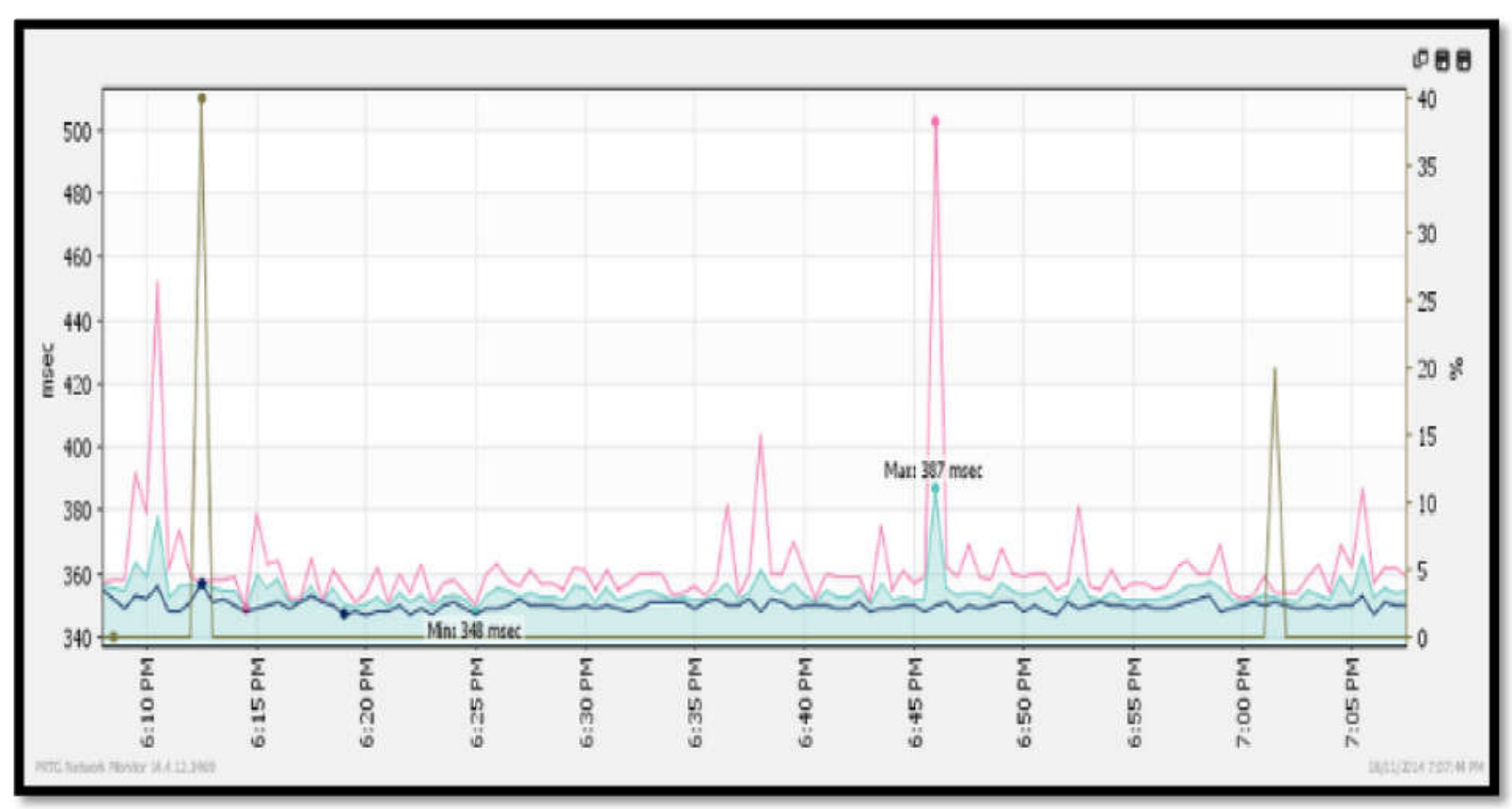

Fig.3. VoIP packet transmission using GSM Codec

In this study, two scenarios have been tested on SIP server performance located at Singapore country (refer to Fig. 4 and Fig. 5). Based on Fig. 4 and Fig. 5, VoIP user from Malaysia has used SIP server located at Singapore. The conversation occurred between $4 \mathrm{pm}$ to $7 \mathrm{pm}$. From the results, it shows that more than $10 \%$ packet loss during the conversation. It is indicated that VoIP conversation have achieved medium to low VoIP quality. Based on ITU standard, good VoIP quality conversation must achieve below than 10\% packet loss. 


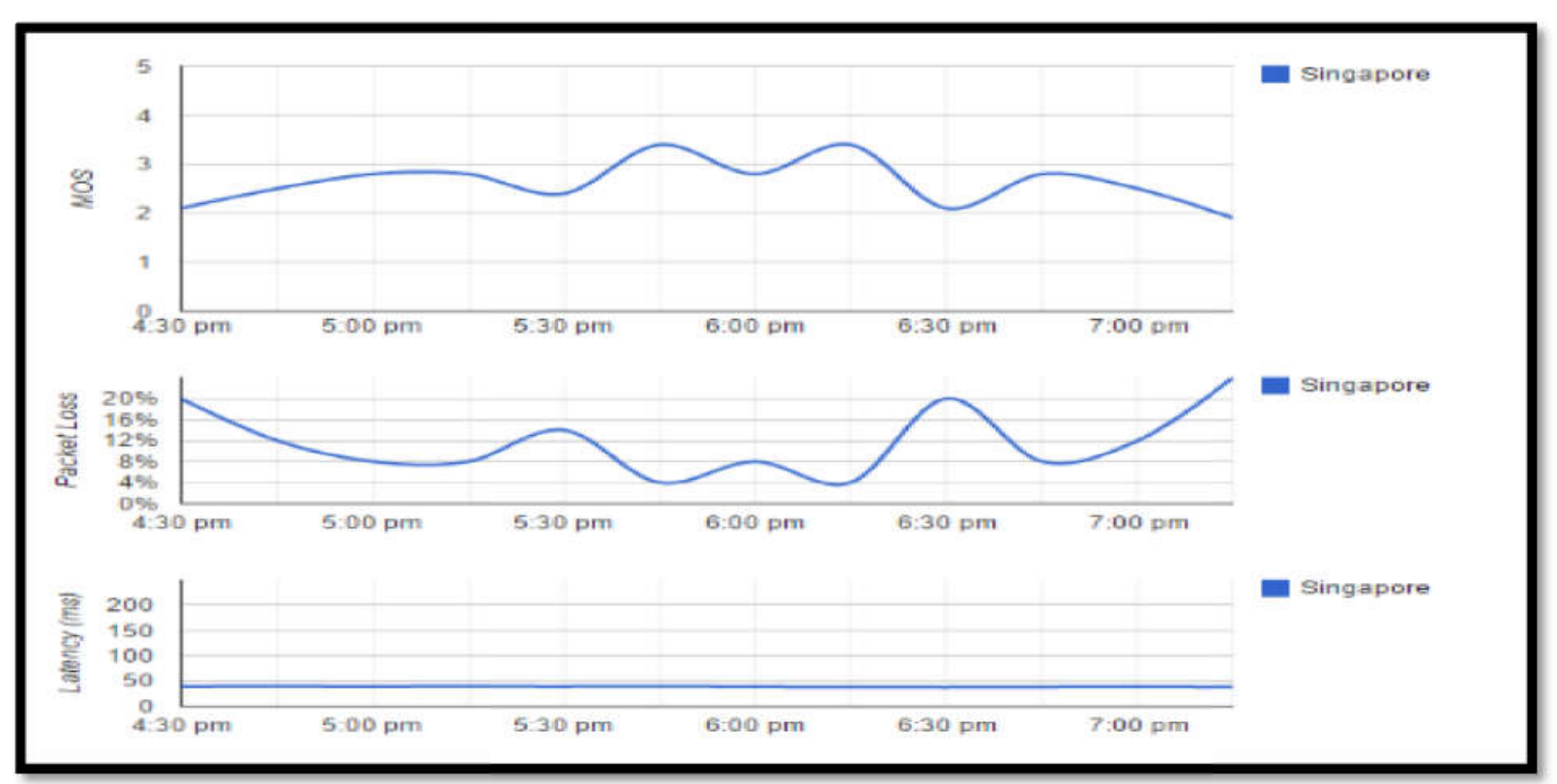

Fig.4. Analysis 1-VoIP conversation from $4.30 \mathrm{pm}$ to $7 \mathrm{pm}$

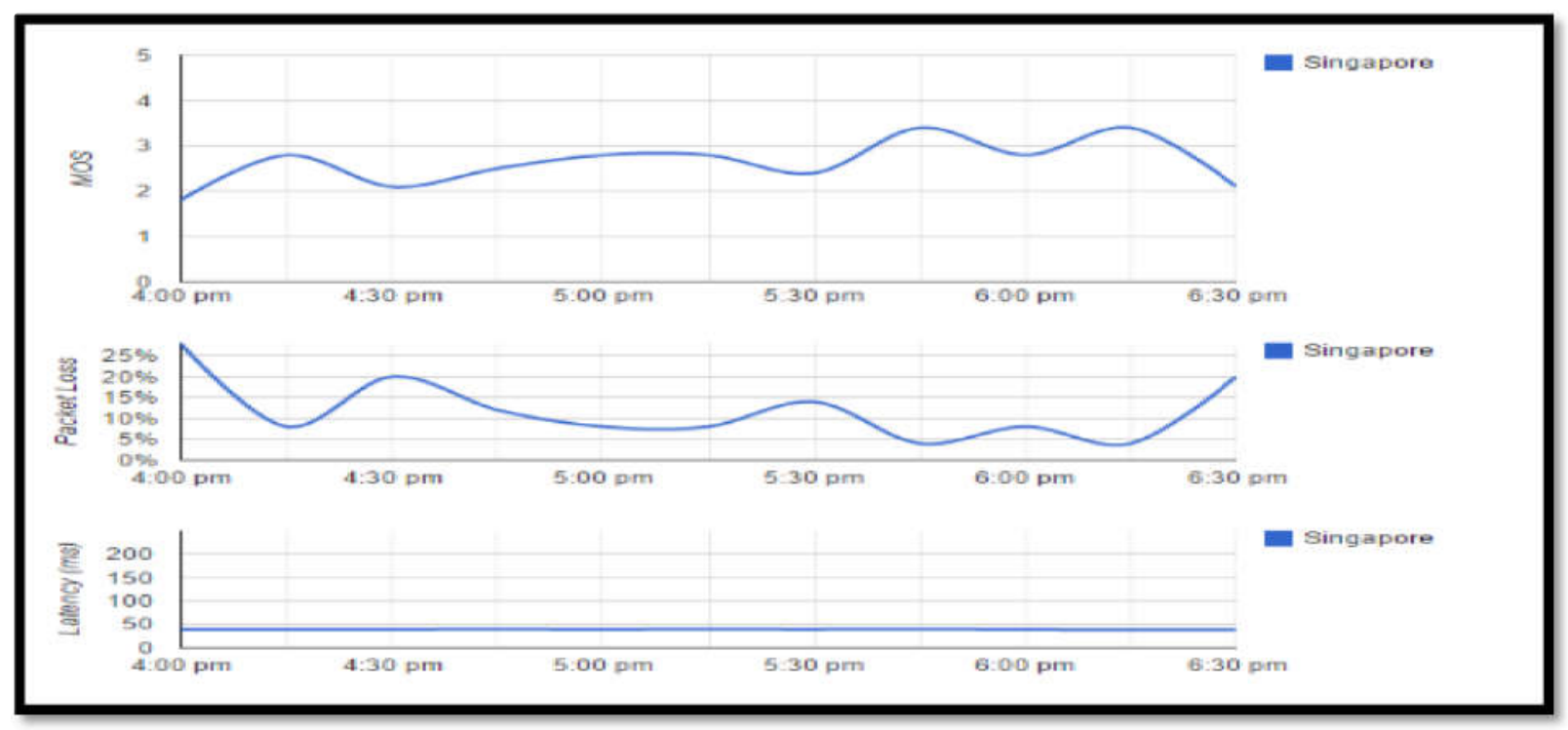

Fig.5. Analysis 2-VoIP conversation from $4 \mathrm{pm}$ to $8.30 \mathrm{pm}$

\section{EXPERIMENTAL}

Fig. 6 below show the network diagram of the project. There are five users in the project that are installed with Linphone software each. Since Linphone offers its own SIP account, we do not have to have personal server to configure the SIP account. The internet connection used in this project is via TM Unifi that is owned by one of our group member. One of the member's laptop is used to run the monitoring tool in order to measure the performance of the codecs involved, refer to Fig. 6 and Fig. 7. 


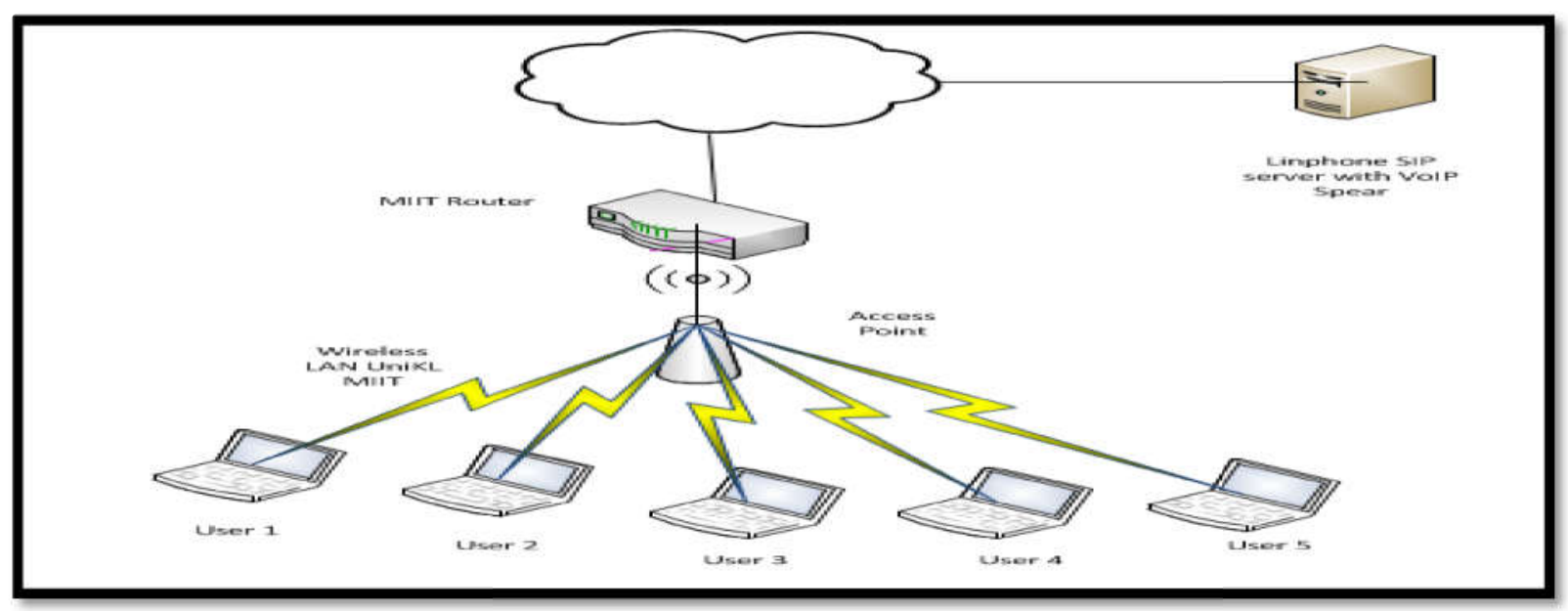

Fig.6. V2oIP technology

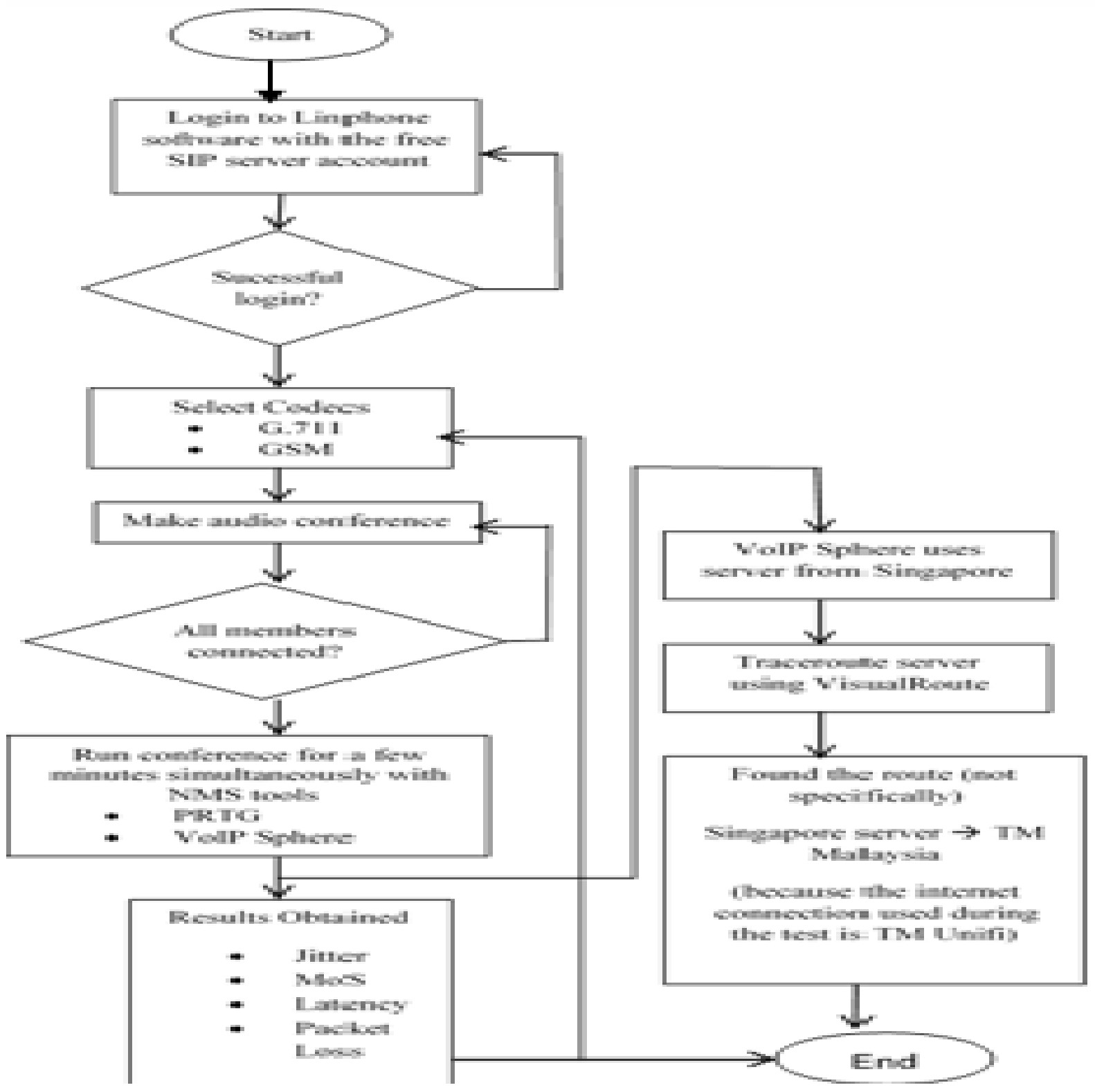

Fig.7. Flowchart of VoIP activities analysis 


\section{CONCLUSION}

Based on the results, using G.711, G.722, iLBC, Speex and G.726 codec are not able to generate high quality of VoIP conversation over wireless carrier network. The result shows that GSM codecs is able to increase VoIP conversation performance over wireless carrier network over Wide Area Network. For VoIP over wired carrier network, the voice quality is better than VoIP over wired carrier network.From the result, we can conclude that VoIP over wireless carrier network has produce a lot of interferences.

There are several factors that can degrade VoIP quality transmission over wireless carrier network as follow: a) queuing delay; b) serialization delay; c) propagation delay; d) transmission delay; and e) codec delay. Since the manual/human MOS tests are quite subjective and less than productive in many ways, there are nowadays a number of software tools that carry out automated MOS testing in a VoIP deployment. Future work, we will extend our experiment on mesh wireless equipment in campus environment.

Future work, the study will focus on several techniques and to analyze the performance of as follows: i) queuing; ii) congestion avoidance; iii) header compression; iv) RSVP; and v) fragmentation. These suggested techniques might be able to increased VoIP throughput in wireless Ipv6 protocol environment such as: i) Compression: Reduces serialization delay and bandwidth required to transmit data by reducing the size of packet headers or payloads; ii) Link Efficiency: Used to improve bandwidth efficiency through compression and link fragmentation and interleaving.

\section{REFERENCES}

[1] Ismail MN. A case study: Server performance measurement for E-learning system in campus environment. Journal Annals. Computer Science Series, Romania, 2011, 9:9-30

[2] Andrusyszyn MA, van Soeren M, Laschinger HS, Goldenberg D, DiCenso A. Evaluation of distance education delivery methods for a primary care nurse practitioner program. International Journal of E-Learning andDistance Education, 2007, 14(1):21-38

[3] Bale E. The rights stuff: Ownership in the digital academy.LearningTechnologies Report, Fall 1999, Volume 1 Number 2. London, Canada: TheNode Learning Technologies Network 
[4] Thompson H. The impact of technology and distance education: A classical learning theory viewpoint. Education Technology and Society,1999, 2(3):25-40

[5] University System of Maryland (USM). Models of distance education. Baltimore: USM, 1997

[6] University of Idaho (UI). Distance education at a glance.Moscow: UI, 2003

[7] Koppelman H,Vranken H. Experiences with a synchronous virtual classroom in distance education. ACM SIGCSE Bulletin, 2008, 4(3):194-198

[8] Massoth M,Korn R. The IP multimedia subsystem with an e-learning management system and integrated video conferencing. In 10th International Conference on Information Integration and Web-Based Applications and Services, 2008, pp. 655-658

[9] Roesler V,Husemann R, Costa C H. A new multimedia synchronous distance learning system: The IVA study case. In ACM Symposium on Applied Computing, 2009, pp. 1765-1770

[10] Sawai S, Donyaprueth K, Nicholas J D, Paul H. The study of e-learning technology implementation: A preliminary investigation of universities in Thailand. Education and Information Technologies, 2006, 11(2):137-160

\section{How to cite this article:}

Ismail M N, Shukran M A, Maskat K, Isa M R M, Ishak S, Sameon S S. Voice over ip using sip server: wide area network performance analysis. J. Fundam. Appl. Sci., 2017, 9(3S), 637-644 